\title{
IMPLICAÇÕES DAS TECNOLOGIAS HÍBRIDAS DE FABRICAÇÃO NOS PROCESSOS DE REPARO E REMANUFATURA DE PEÇAS NO CONTEXTO DA MANUTENÇÃO: UMA REVISÃO SISTEMÁTICA
}

\author{
IMPLICATIONS OF HYBRID MANUFACTURING TECHNOLOGIES ON PARTS REPAIR AND \\ REMANUFACTURING PROCESSES IN MAINTENANCE CONTEXT: A SYSTEMATIC REVIEW
}

Daniel R. Tasé Velázquez ${ }^{1}$

André Luís Helleno²

Carlos Roberto Camello Lima ${ }^{3}$

Lorena Hernández Mastrapa ${ }^{4}$

${ }^{1}$ Mestrado em Metrologia (2017)

pelo Programa de Pós-graduação em Metrologia - Pós MQI da Pontifícia

Universidade Católica do Rio de Janeiro, Brasil. Doutorando em Engenharia de Produção (2018-2022) no Programa de Pós Graduação em Engenharia de Produção da Universidade Metodista de

Piracicaba

Brasil.

dtasev88@gmail.com

2Doutorado em Engenharia de Produção (2008) pela Universidade Metodista de Piracicaba, Brasil. Professor Assistente da Escola de Engenharia da Universidade Presbiteriana Mackenzie, Brasil.

ahelleno@gmail.com

${ }^{3}$ Doutorado em Engenharia Mecânica (1996) pela Universidade Estadual de Campinas, Brasil.

Professor Assistente da Escola de Engenharia

da Universidade Presbiteriana Mackenzie, Brasil ccamellolima@gmail.com

\footnotetext{
${ }^{4}$ Mestrado em Engenharia de Produção (2017) pelo Programa de Pós-graduação em Engenharia de Produção da Pontifícia

Universidade Católica do Rio de Janeiro, Brasil. Doutoranda em Engenharia de Produção (2017-2021) no Programa de Pós Graduação em Engenharia de Produção da Universidade Metodista de Piracicaba

Brasil.

lorenahmastrapa@gmail.com
}

\begin{abstract}
Resumo
A flexibilidade das tecnologias híbridas de fabricação (HMT) para integrar os processos produtivos e as atividades de manutenção contribui para o aumento do ciclo de vida de peças funcionais ou remanufaturadas, agregando-lhes valor, dado que, pelo elevado custo de fabricação, não se justifica seu descarte. O objetivo deste artigo é investigar quais são os benefícios do uso das HMT nas atividades de reparo e remanufatura de peças no contexto da manutenção. A revisão sistemática da literatura relacionada ao tema mostrou que o emprego das HMT para executar atividades de reparo e remanufatura de peças como parte dos sistemas de manutenção está em expansão. Além disso, foi identificado que as aplicações das HMT visam o aumento do ciclo de vida dos produtos, a eficiência de recursos e remanufatura de peças no fim da vida útil como parte da reconfiguração da cadeia de valor.
\end{abstract}

Palavras-chave: Tecnologias híbridas de fabricação. Manufatura aditiva e subtrativa. Manutenção, reparo, remanufatura.

\begin{abstract}
The flexibility of hybrid manufacturing technologies (HMT) to integrate production processes and maintenance activities contributes to increasing the lifecycle of functional or remanufactured parts, adding value to them, given that the high manufacturing cost, do not justifies its disposal. The purpose of this article is to investigate the benefits of using HMT for repairing and remanufacturing parts in maintenance context. The systematic review of literature related to the topic showed that the use of HMT to perform parts repair and remanufacturing as part of maintenance systems is expanding. In addition, it has been identified that HMT applications aim to increase the product lifecycle, resource efficiency and end-of-life part remanufacturing as part of the value chain reconfiguration.
\end{abstract}

Keywords: Hybrid manufacturing technologies. Additive and subtractive manufacturing. Maintenance, repair, remanufacturing. 


\section{Introdução}

As tecnologias híbridas de fabricação (HMT) que combinam processos de manufatura aditiva (AM - additive manufacturing) (Flynn et al., 2016; Newman et al., 2016) e processos de fabricação subtrativos (SM - subtractive manufacturing processes) (Zhu et al., 2013; Liu et al., 2017), como por exemplo, o processo de usinagem (Zębala \& Plaza, 2014), apresentam vantagens competitivas superiores em relação aos modelos tradicionais de fabricação, no que se refere a: melhor utilização dos recursos humanos, materiais e tecnológicos, assim como a redução do time-to-market, tempos de ciclo, e o lead time das peças e componentes produzidos (Karunakaran et al., 2010; Guessasma et al., 2015; Attaran, 2017). Isso proporciona às empresas uma elevada flexibilidade e agilidade na mudança de roteiros de fabricação (Faludi et al., 2015). A natureza dupla destes sistemas permite iniciar o processo de fabricação empregando, tanto o processo aditivo, quanto o subtrativo.

O escopo das HMT inclui a fabricação de peças com superfícies complexas que, naturalmente, não poderiam ser construídas pelos métodos convencionais de manufatura devido à pouca acessibilidade das ferramentas de usinagem a diferentes áreas da peça (Lasemi et al., 2010; Xiao et al., 2018), e a remanufatura de peças a partir de componentes já existentes, reduzindo a quantidade de material utilizado, diminuindo o tempo e custos de fabricação, e aumentando a flexibilidade no processamento (Flynn et al., 2016; Newman et al., 2016; Liu et al., 2017).

A necessidade da combinação de técnicas aditivas e subtrativas surge devido a que peças metálicas fabricadas ou remanufaturadas por meio da AM requerem posterior processamento para refinar sua exatidão geométrica (forma) e melhorar a qualidade da superfície (Guessasma et al., 2015; Newman et al., 2016; Liu et al., 2017). Métodos convencionais de fabricação para o acabamento de peças metálicas podem ser vantajosos devido à facilidade de integração entre software e hardware, e a capacidade de processar o material seletivamente (Singh et al., 2017).

O processo híbrido é aplicado com sucesso no reparo e remanufatura de peças metálicas funcionais ou a partir de componentes já existentes, agregando valor a peças que, pelo elevado custo de fabricação, não justificam seu descarte. Tanto na produção, quanto no reparo/remanufatura de peças e componentes, as HMT garantem uma elevada confiabilidade metrológica, atendendo aos requisitos de qualidade exigidos para algumas aplicações, como, por exemplo, na indústria de construção, esportiva e automotiva (Tofail et al., 2018).

Liu et al. (2017) afirmaram que o emprego das HMT cria oportunidades significativas na fabricação de peças novas e na remanufatura de peças desgastadas ou danificadas. Neste sentido, Newman et al. (2016) indicaram que os sistemas híbridos podem ser usados na reparação de peças de metal, usinando primeiramente a zona danificada, depositando material logo em seguida onde requerido e depois usinando a superfície onde o material foi depositado, para refinar a geometria e as características da superfície. Flynn et al. (2016) afirmaram que esta abordagem de reparação por 
adição e subtração de material situa-se naturalmente dentro de setores que produzem partes de baixo volume e alta complexidade, que estão sujeitas a desgaste e danos críticos, como nas indústrias aeroespacial e aeronáutica, militar, médica, entre outras.

Com o destaque e a popularização do emprego das HMT no setor industrial, surgiu a necessidade de desenvolver e certificar processos e sistemas de manutenção com reparo e remanufatura empregando estas tecnologias (Ford \& Despeisse, 2016). Este trabalho se propõe a investigar os benefícios do uso das HMT para reparo e remanufatura de peças no contexto das atividades de manutenção. Foi desenvolvida uma revisão sistemática sobre o tema, com o objetivo de identificar o estado da arte das pesquisas neste campo do conhecimento, por meio de um mapeamento dos trabalhos de interesse, assim como sintetizar as principais contribuições neste assunto.

No intuito de homogeneizar alguns dos conceitos que fazem parte deste trabalho, indicam-se, a seguir, algumas definições.

Na ABNT NBR 5462:1994, o termo manutenção é definido com a "combinação de todas as ações técnicas e administrativas, incluindo as de supervisão, destinadas a manter ou recolocar um item em um estado no qual possa desempenhar uma função requerida". Nota: A manutenção pode incluir uma modificação do item. Conforme Pinto \& Xavier (2009), define-se a manutenção como a garantia da disponibilidade da função dos equipamentos e instalações, de modo a atender um processo de produção ou serviço, com confiabilidade, segurança, preservação do meio ambiente e custos adequados.

O reparo abrange a correção de falhas identificadas em um produto (King et al., 2006). Já a remanufatura é o processo em que os produtos usados são devolvidos, após uma intervenção, como mínimo para as especificações de desempenho dos fabricantes originais de equipamentos (OEM original equipment manufacturer), desde o ponto de vista do cliente e, ao mesmo tempo, são dadas garantias iguais às dos novos produtos equivalentes (ljomah, 2002).

\section{Método de pesquisa}

Para coletar e analisar os principais documentos científicos publicados relacionados com o objeto de estudo deste trabalho, foi estruturado um método de quatro etapas, adaptado de Fahimnia et al. (2015).

Etapa I - Definição das palavras-chave: para direcionar a pesquisa e reunir os trabalhos mais relevantes, foram identificadas 12 palavras-chave, a partir da revisão de trabalhos relacionados com as áreas de estudo, de forma independente. As palavras-chave agruparam-se segundo cada contexto de pesquisa como apresentado no Quadro 1. 
Quadro 1 - Palavras-chave definidas no contexto das áreas de pesquisa

\begin{tabular}{|c|c|}
\hline Contexto & Palavras-chave \\
\hline Manufatura híbrida & $\begin{array}{l}\text { Hybrid manufacturing, hybrid additive-subtractive manufacturing, additive } \\
\text { manufacturing, 3D printing, three dimensional printing, rapid prototyping, CNC } \\
\text { machining, subtractive manufacturing, rapid tooling. }\end{array}$ \\
\hline Manutenção & Maintenance, remanufacturing, repair. \\
\hline
\end{tabular}

Fonte: Elaborado pelos autores.

Etapa II - Estratégia de busca e protocolo: a busca bibliográfica foi feita delimitando os seguintes strings: (i) "hybrid additive-subtractive manufacturing" AND "maintenance" AND "repair"; e (ii) "hybrid additive-subtractive manufacturing" AND "maintenance" AND "remanufacturing". Cada string implicou uma busca de forma independente. Os termos restantes no contexto da manufatura híbrida foram usados como critérios qualificadores de inclusão de trabalhos relacionados, com o fim de refinar a busca. Outros critérios como idioma, período de publicação, tipo de material, tópico (relacionado ao resto das palavras chaves), recursos, etc., também foram empregados. O protocolo de pesquisa que foi estruturado é apresentado no Quadro 2.

\section{Quadro 2 - Protocolo de pesquisa}

Protocolo de pesquisa

string i: "hybrid additive-subtractive manufacturing" and "maintenance" and "repair";

string ii: "hybrid additive-subtractive manufacturing" and "maintenance" and "remanufacturing"

Filtros qualificadores de inclusão (I) e exclusão (E)

(I) artigos com presença dos termos (strings) exatos, em qualquer parte do documento, incluindo o título, resumo e palavras-chave.

(I) artigos com período de publicação: 2010-2019.

(I) tipo de material: artigos científicos.

(I) recursos: apenas artigos revisados por pares.

(I) artigos com contribuição teórica ou prática, identificada por meio de triagem manual após a análise de conteúdo.

(I) artigos de interesse achados pela técnica snowballing.

(E) artigos duplicados.

(E) excluir livros.

(E) trabalhos fora do idioma inglês.

(E) artigos cujo escopo não esteja relacionado a: "engineering", "3d printing", "maintenance" "rapid prototyping", "hybrid manufacturing", "repair", "remanufacturing" "cnc machining", "subtractive manufacturing"

(E) artigos fora do escopo da pesquisa (publicações comerciais, industrial viewpoints, etc.).

Fonte: Elaborado pelos autores. 
Etapa III - Seleção das bases de dados: a pesquisa bibliográfica foi feita no Portal de Periódicos da CAPES (Coordenação de Aperfeiçoamento de Pessoal de Nível Superior). A busca permitiu extrair todos os documentos, ordenados por relevância, com presença dos termos de pesquisa independentemente da base de dados onde estava indexado o trabalho. Os trabalhos de interesse agrupados foram extraídos das bases de dados Science Direct (Elsevier), Springer Link, Semantic Scholar, Elsevier (CrossRef), SAGE Journals online, ASME Digital Collection, CiteSeerx, StudyLib, Emerald Insight, Wiley Online Library, GALE Academic OneFile e Inderscience. No caso desta última, que não está indexada ao portal CAPES, foi consultada para acessar a trabalhos encontrados pela técnica de snowballing.

Etapa IV - Análise dos dados: uma abordagem indutiva (Miguel, 2012) foi usada para formular critérios conclusivos a partir dos resultados do processamento bibliométrico. Como principiais resultados apresenta-se uma análise documental preliminar dos trabalhos coletados por meio da aplicação do protocolo estruturado, o estudo bibliométrico, que inclui: visualização das palavras-chave, tendência de publicações, principais veículos de publicação, análise dos autores, e representatividade internacional. Já a análise de conteúdo realizada permitiu identificar as abordagens práticas dos artigos, que foram agrupados em uma tabela segundo a semelhança da abordagem estudada no contexto da pesquisa. Como ferramenta principal para o desenvolvimento da bibliometria, o software para gerenciamento de referências Mendeley foi usado para extrair os dados bibliográficos dos artigos. Estes dados foram agrupados e exportados em um arquivo .RIS e, posteriormente processados no software BibExcel. Além disso, outro arquivo.XML foi gerado usando o Mendeley e exportado para uma planilha, com o fim de complementar o processamento bibliométrico. Foi utilizado o software Gephi para representar uma rede de colaboração dos autores/coautores com mais publicações. As palavras-chave identificadas nos trabalhos podem ser visualizadas em uma figura que as agrupa segundo a frequência de menção.

\section{Resultados e discussões}

\subsection{Análise documental preliminar}

A revisão sistemática da literatura foi desenvolvida até fevereiro de 2019. A partir dos dois strings de busca, foram obtidos 540 documentos. Essa quantidade foi reduzida gradualmente aplicando filtros de inclusão/exclusão, limitando a quantidade a apenas aquela que continha os termos exatos presentes em qualquer parte dos documentos, publicados nos últimos 10 anos (20102019), considerando apenas artigos científicos no idioma inglês, excluindo livros. Nos tópicos de pesquisa gerados pelo portal de busca, adotaram-se como critérios de exclusão aqueles artigos que não se relacionavam com as restantes palavras-chave associadas a cada contexto. Isso permitiu agrupar 218 artigos de interesse. Aplicando outros critérios de exclusão, como "trabalhos revisados 
por pares", e eliminando os que não estavam dentro do escopo da pesquisa (publicações comerciais e industrial point-of-view), revisados por meio de uma triagem manual para analisar o título, resumo, palavras-chave e contribuição teórico-prática no texto do artigo e técnica de snowballing, foi agrupado um total de 37 artigos, que se considera pertinentes à pesquisa. Desses 37, sete artigos (anteriores a 2010) foram encontrados aplicando o snowballing. O protocolo de pesquisa, que inclui os critérios de busca, filtros de inclusão/exclusão, assim como a quantidade de artigos resultantes, é apresentado na Tabela 1.

Tabela 1 - Protocolo de pesquisa e quantidade de artigos resultantes

$$
\text { Protocolo de pesquisa }
$$

Quantidade de documentos

strings: "hybrid additive-subtractive manufacturing" and "maintenance" and "repair"; "hybrid additive-subtractive manufacturing" and "maintenance 540 implications" and "remanufacturing". Filtros de Inclusão (I) / Exclusão (E)

(I) Artigos com presença dos termos (strings) exatos, em qualquer parte do documento, incluindo o título e o resumo; (I) Artigos com período de publicação: últimos 10 anos; (I) Tipo de material: artigos científicos.

(I) Recursos: apenas artigos revisados por pares; (E) Artigos duplicados; (E) Excluir livros; (E) Trabalhos fora do idioma inglês; (E) Artigos fora do escopo relacionado a: "engineering", "3d printing", "maintenance" "rapid prototyping", "hybrid manufacturing", "repair", "remanufacturing" "cnc machining", "subtractive manufacturing" Outros critérios de Inclusão (I) / Exclusão (E)

(E) publicações comerciais, industrial point-of-view, etc.; (I) Artigos com contribuição teórica ou prática, identificada por meio de triagem manual após a análise do título, resumo, palavras-chave e de conteúdo; (I) Artigos fora do período especificado, mas, que sejam relevantes no contexto da pesquisa (snowballing).

Fonte: Elaborada pelos autores.

Os resultados preliminares da análise da literatura, seguindo os procedimentos metodológicos definidos neste trabalho, permitem concluir que os trabalhos científicos publicados sobre as implicações da manufatura híbrida para reparo e remanufatura de peças no contexto das atividades de manutenção, ainda são limitados (37 documentos), apesar da relevância e novidade do tema. Isto pode ser condicionado devido à pouca ou limitada acessibilidade à estrutura industrial necessária para implantar esse tipo de modelo de fabricação, o que, por consequência, limita as pesquisas relacionadas aos avanços na área, dado que esta é uma área de estudo relativamente nova. 


\subsection{Visualização das palavras-chave}

As palavras-chave de cada artigo foram extraídas usando o Mendeley, e a frequência controlada pelo BibExcel. No total, 154 palavras-chave foram identificadas. Na Figura 1, apresentamse, para melhor visualização, as palavras-chave mencionadas com maior frequência nos artigos, totalizando 86 .

Figura 1 - Palavras-chave por frequência de menção com base no Mendeley

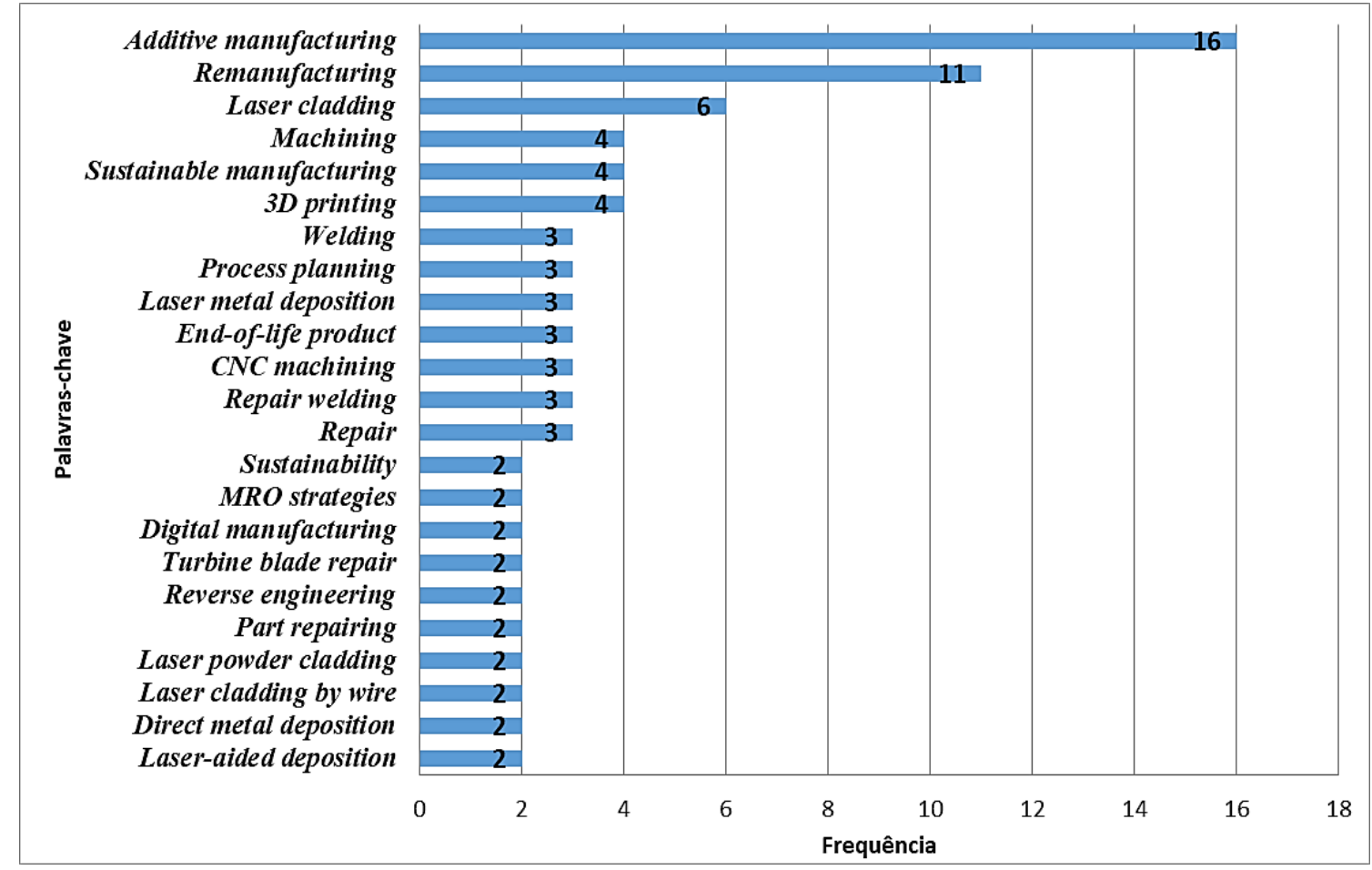

Fonte: Elaborada pelos autores.

Percebe-se que o termo "remanufatura" aparece com frequência igual a 11; já os termos "reparo" e "reparo de peças" aparecem mencionados três e duas vezes, respectivamente. O termo "manutenção", que não está referenciado na figura, aparece apenas uma vez. Outras 28 palavraschave de interesse foram extraídas em função da relevância destes termos na pesquisa e se listam no Quadro 3. Estas palavras-chave podem ser empregadas em pesquisas bibliográficas futuras. 
Quadro 3 - Palavras-chave relevantes com frequência=1

\begin{tabular}{|l|l|}
\hline Palavras-chave com frequência=1 & \\
\hline Blade repair & Life cycle engineering \\
\hline Direct material reuse & Maintenance \\
\hline Direct metal laser sintering & Metal Part Repair \\
\hline Environmentally conscious manufacturing & Part remanufacturing \\
\hline Hybrid additive manufacturing & Product life cycle \\
\hline Hybrid manufacturing & Repair and overhaul \\
\hline Hybrid manufacturing processes & Repair technology \\
\hline Hybrid processing & Repairing welding \\
\hline Industrial sustainability & Resource efficiency \\
\hline Inspection & Subtractive manufacturing \\
\hline Laser remanufacturing & Circular economy \\
\hline Life cycle & Tool repair \\
\hline Life cycle analysis & Value chain reconfiguration \\
\hline Life cycle assessment & Waste \\
\hline
\end{tabular}

Fonte: Elaborado pelos autores.

De forma geral, a mineração das palavras-chave apresenta os temas-chave das pesquisas referentes a, aumento da ciclo de vida dos produtos; eficiência de recursos por meio da eliminação de desperdício, relacionando-se de forma estreita com a sustentabilidade; remanufatura de peças no fim da vida útil como parte da reconfiguração da cadeia de valor, etc. A análise visual permite identificar os principais processos aditivos e subtrativos empregados. No primeiro caso, os processos aditivos de revestimento a laser e deposição de material direta a laser; no segundo, processos subtrativos baseados na usinagem.

\subsection{Tendência de publicações}

A partir da análise dos 37 artigos agrupados, foi possível identificar a tendência de publicação por ano. A Figura 2 apresenta esta tendência. 
Figura 2 - Tendência de publicações por ano

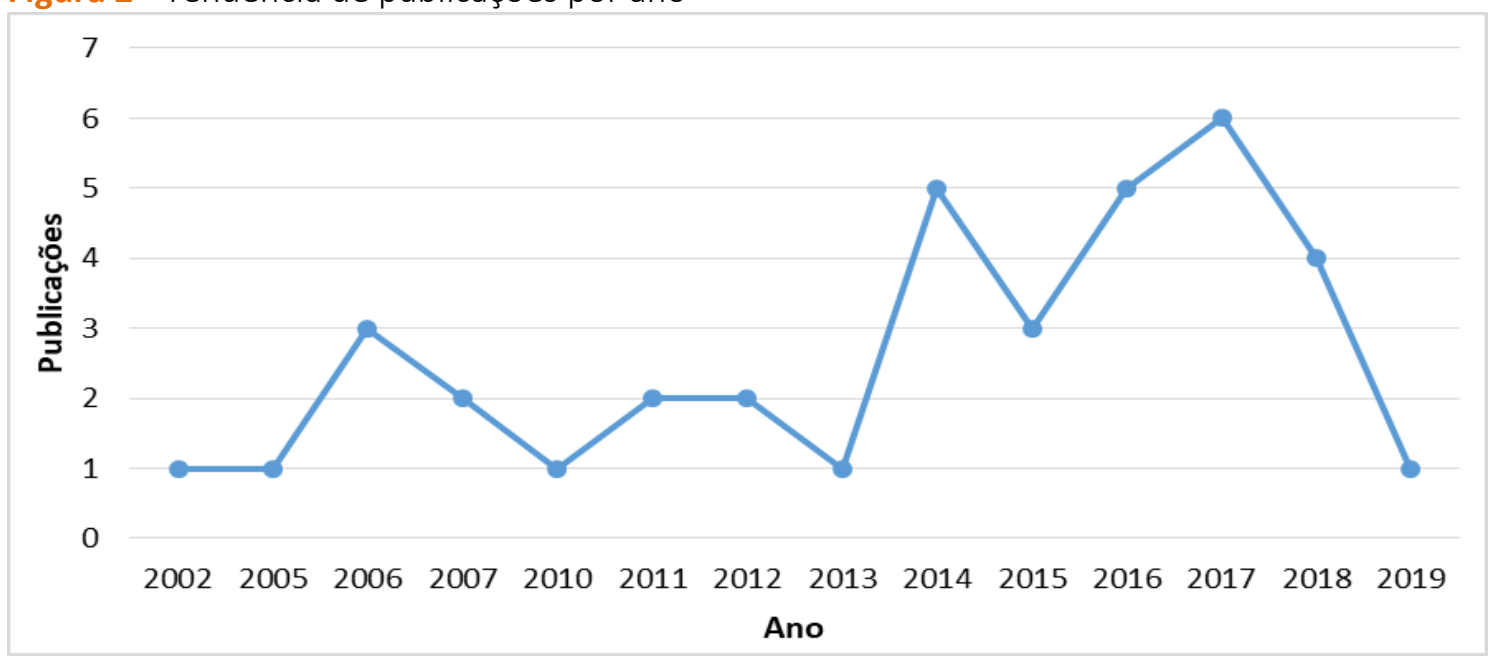

Fonte: Elaborada pelos autores.

Observa-se, na Figura 2, que, a partir de 2002, até o período de revisão em 2019, existe uma tendência leve de aumento dos trabalhos científicos, embora de 2006 a 2010, de 2014 a 2015 e de 2017 a 2019, diminuísse. O pico máximo de publicações (6) aparece em 2017, representando aproximadamente $16 \%$ do total do período. Infere-se que o fato de as publicações serem poucas pode ser consequência da consolidação dos métodos tradicionais de fabricação na indústria e do incipiente conhecimento teórico e prático da academia sobre as implicações do emprego das HMTs como modelo avançado de manufatura. No relatório do estudo desenvolvido pelo Institute for Defense Analyses dos Estados Unidos da América (IDA, 2012), identificou-se a AM como uma das tendências emergentes no âmbito das tecnologias de manufatura avançada para os próximos dez anos a partir daquele momento e, como cenários futuros, a necessidade do gerenciamento dos riscos associados ao emprego de modelos produtivos cada vez mais complexos, dado o incremento gradual da demanda de bens altamente tecnológicos (high-technology goods). Isto pode ser também consequência do aumento gradual, mas leve, das publicações a partir de 2012.

\subsection{Principais veículos de publicação}

Os 37 documentos foram publicados em 24 veículos diferentes. Os periódicos onde mais artigos foram publicados são o Physics Procedia, com cinco publicações, o Journal of Cleaner Production e o Procedia CIRP, com quatro publicações cada um. A Figura 3 apresenta os cinco veículos onde mais se publicou e a quantidade de artigos respectivos, para um total de 17 trabalhos, representando $46 \%$ do total agrupado. 
Figura 3 - Veículos onde mais se publicou e quantidade de trabalhos por ano

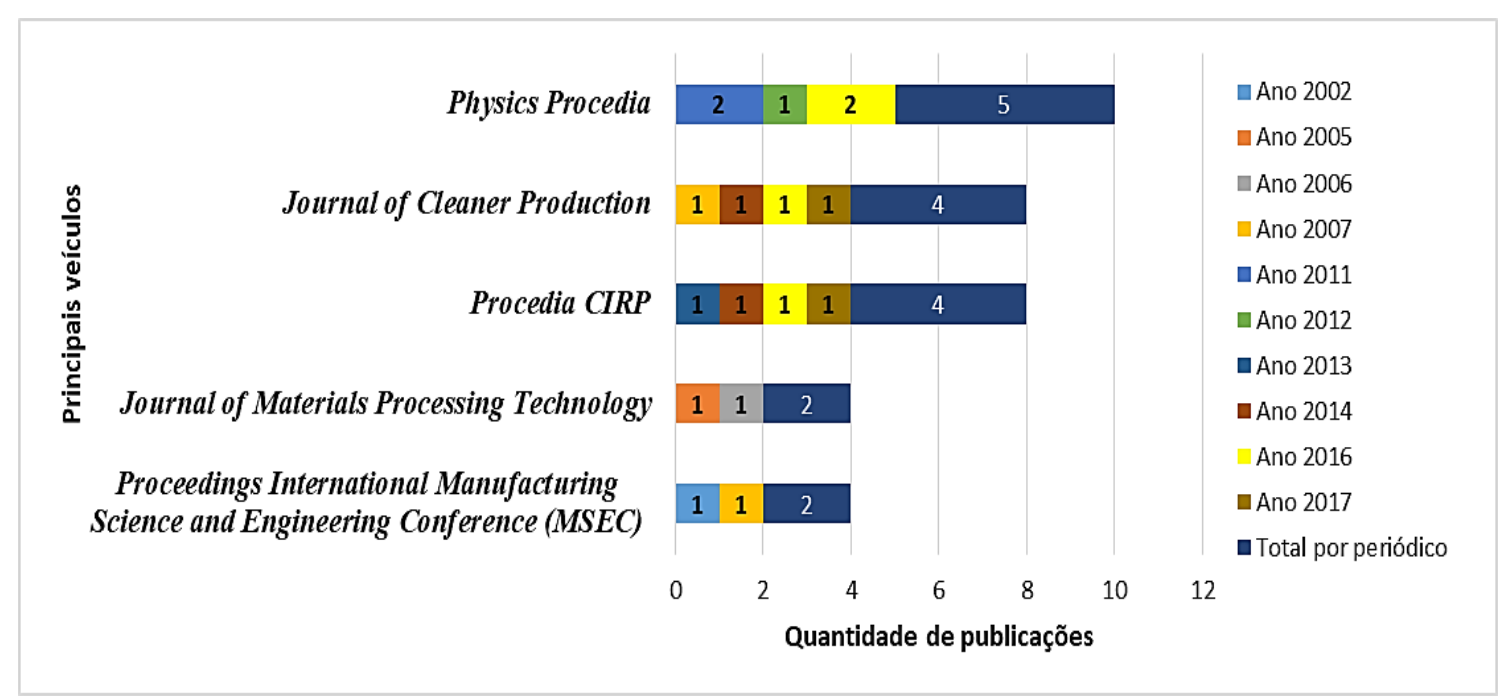

Fonte: Elaborada pelos autores.

\subsection{Análise dos autores}

Foram identificados, no total, 97 autores. Destes, somente 14 aparecem em duas ou mais publicações e, no máximo, em cinco. Na Figura 4, apresentam-se esses autores e a quantidade de publicações respectivas.

Figura 4 - Autores/coautores com mais publicações

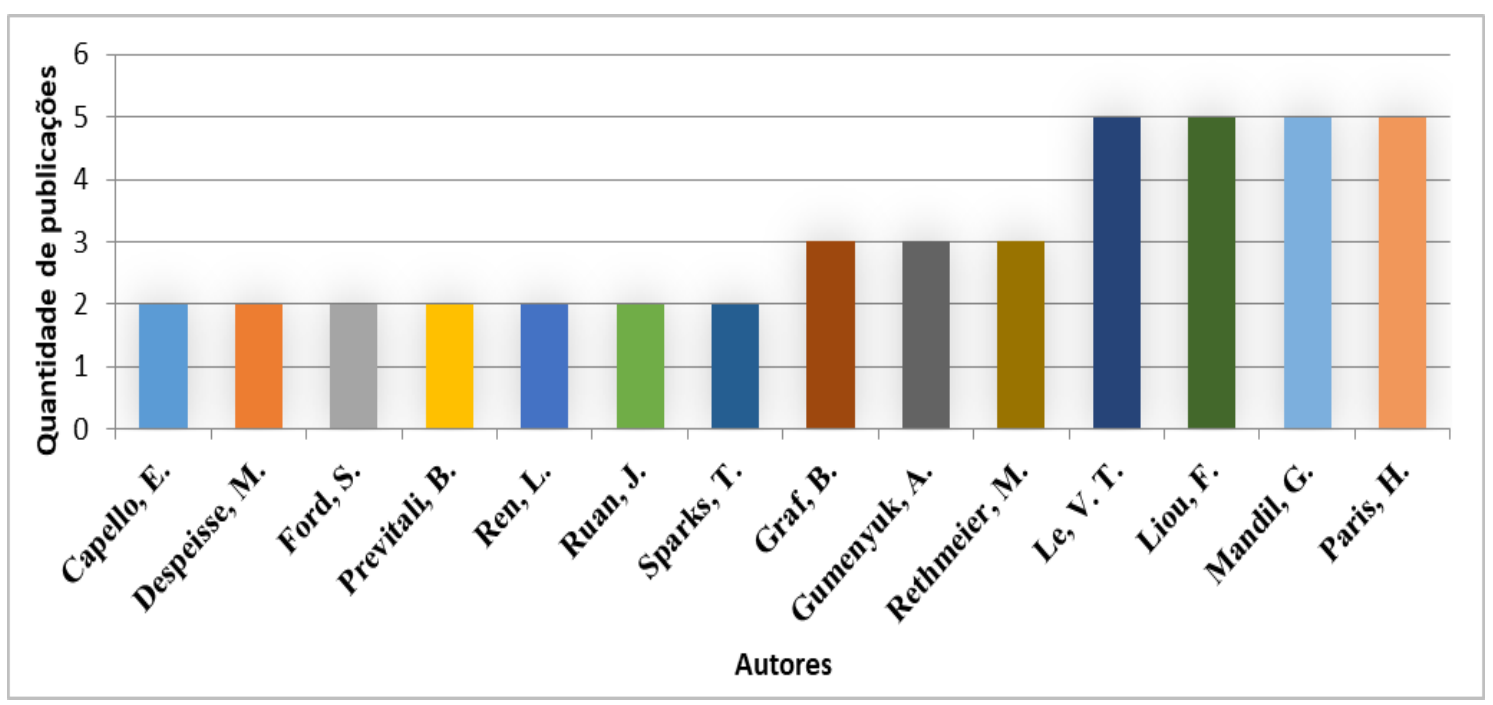

Fonte: Elaborada pelos autores.

Os dados extraídos sobre os autores permitiram observar a existência de uma rede de colaboração entre eles. Por meio do software Gephi, foi construída esta rede, representada na Figura 5. A rede de colaboração representa a relação entre os autores que aparecem em duas ou mais publicações (Figura 4) com outros autores/coautores, mesmo que estes apenas tenham colaborado em uma publicação. 
Figura 5 - Rede de colaboração entre os autores com mais publicações

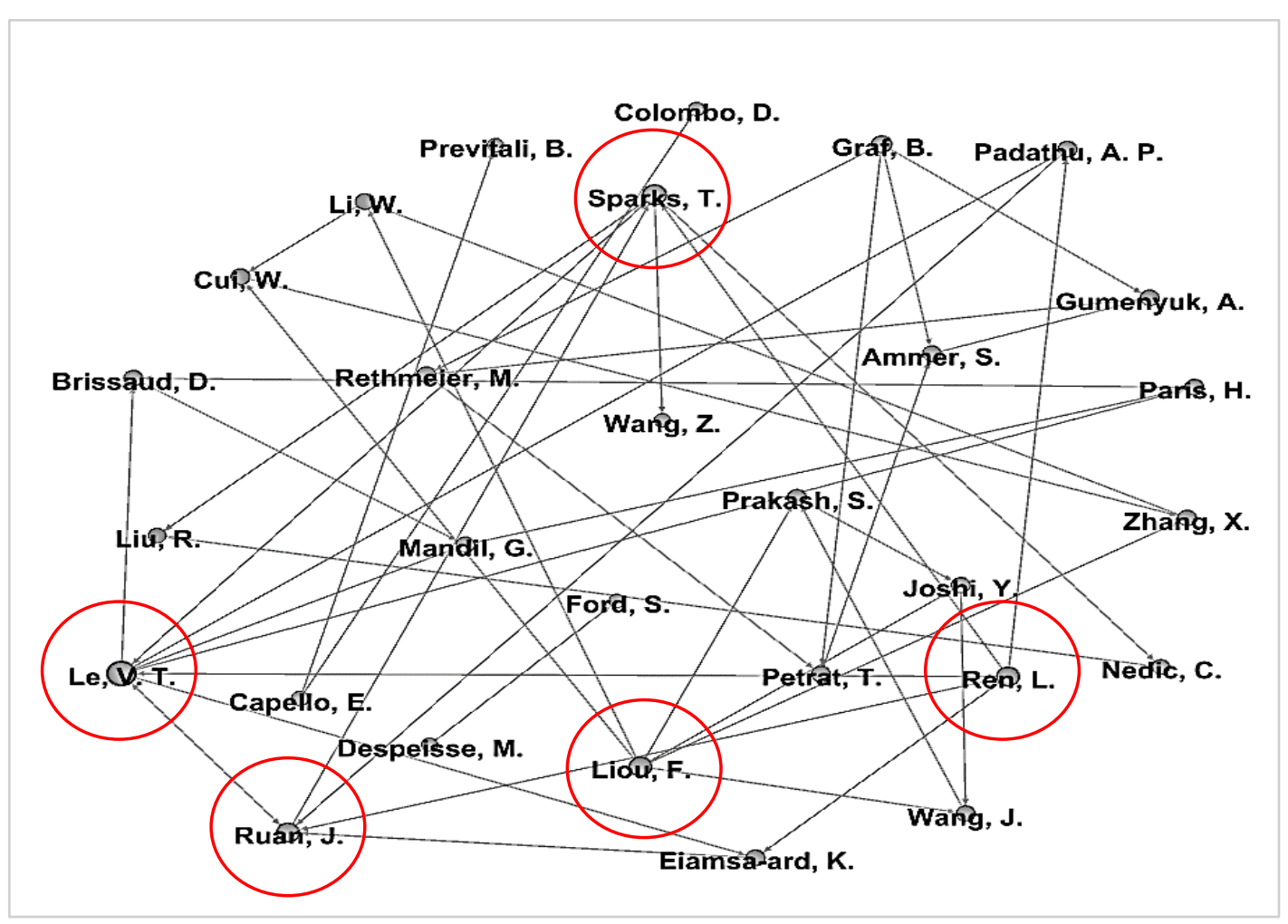

Fonte: Elaborada pelos autores.

Os nós maiores, marcados com círculo vermelho, representam os autores com maior grau de colaboração. No caso, Le, V-T. aparece com maior grau, seguido por Liou, F., Sparks, T., Ren, L e Ruan, J. Um grau de colaboração maior indica que este autor trabalhou com maior número de colaboradores para desenvolver o artigo.

\subsection{Representatividade internacional}

A partir dos dados levantados, foram extraídos os países que contribuem na pesquisa. A Figura 6 indica, em forma decrescente no sentido horário, os países onde há maior representatividade na pesquisa da área de estudo e a quantidade de artigos respectivos. 
Figura 6 - Países com maior contribuição na pesquisa e quantidade de artigos respectivos

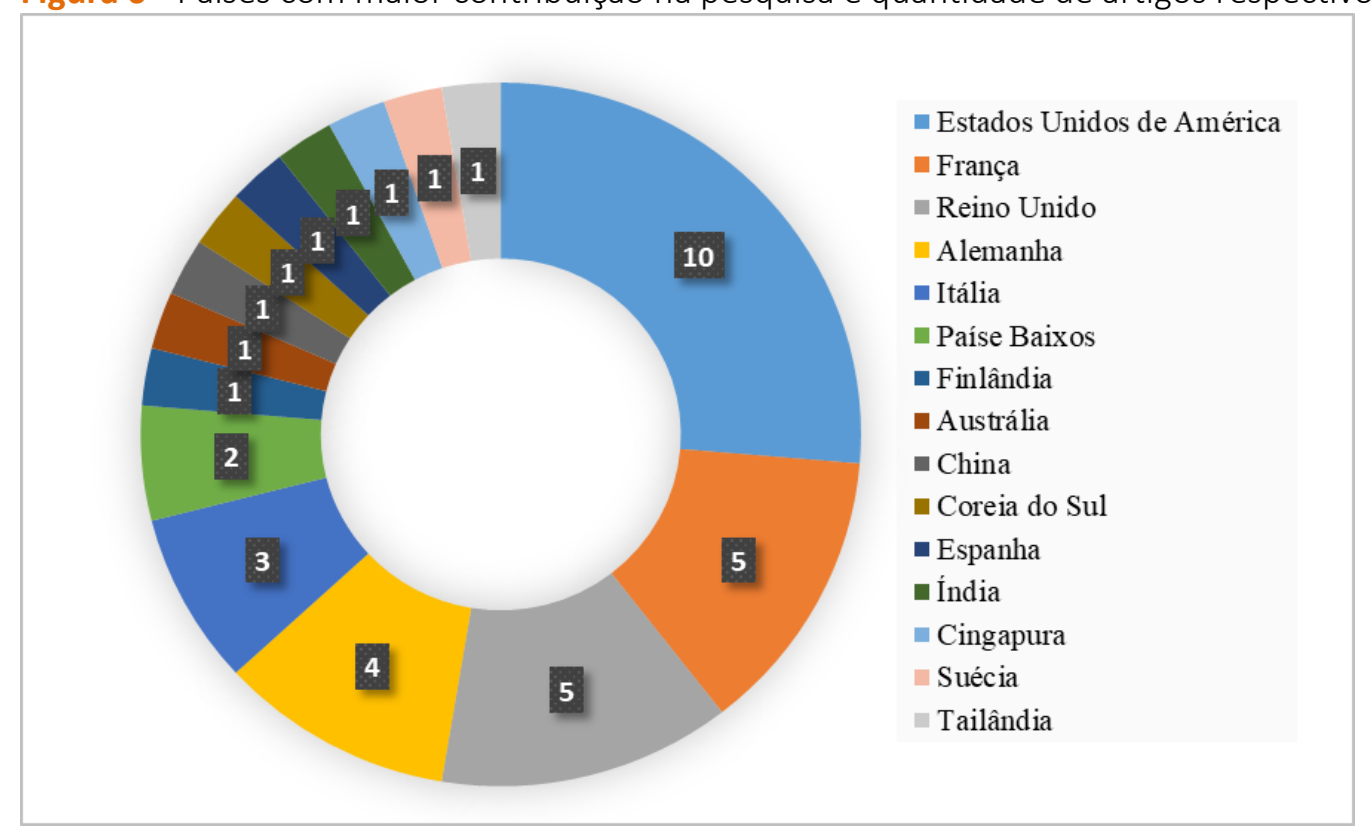

Fonte: Elaborada pelos autores.

No total, 15 países foram os mais destacados. Os países que mais contribuíram na pesquisa segundo a densidade de artigos são: Estados Unidos de América (10), França (5), Reino Unido (5), Alemanha (4), Itália (3), Países Baixos (2) e Finlândia, Austrália, China, Coreia do Sul, Espanha, Índia, Cingapura, Suécia e Tailândia, com apenas um (1) artigo. A soma total dos artigos é maior do que 37, pois em um mesmo artigo participaram autores de distintos países. Consequentemente, pesquisadores afiliados a 8 centros de pesquisa de um total de 37 instituições colaboram com mais de um artigo. Percebeu-se a existência de uma ampla heterogeneidade de instituições envolvidas. Na Tabela 2, apresentam-se as instituições com maior contribuição, segundo a quantidade de artigos, destacando-se as instituições de EUA, França, Alemanha, Reino Unido, Itália e os Países Baixos.

Tabela 2 - Universidades com maior contribuição de publicações por países

\begin{tabular}{|l|c|l|}
\hline Universidades com maior contribuição & Artigos & Países \\
\hline Missouri University of Science and Technology & 5 & EUA \\
\hline Université Grenoble-Alpes & 5 & França \\
\hline Federal Institute for Materials Research and Testing & 3 & Alemanha \\
\hline Fraunhofer Institute for Production Systems and Design Technology & 3 & Alemanha \\
\hline University of Bath & 2 & Reino Unido \\
\hline University of Cambridge & 2 & Reino Unido \\
\hline Politecnico di Milano & 2 & Itália \\
\hline University of Twente & 2 & Países Baixos \\
\hline
\end{tabular}

Fonte: Elaborada pelos autores. 
Na análise das afiliações institucionais, observou-se uma colaboração entre as instituições de Singapura e Índia, e entre EUA e Tailândia. No caso, as duas colaborações mais relevantes são citadas na Tabela 3.

Tabela 3 - Colaborações entre países e artigos respectivos

\begin{tabular}{|l|l|c|}
\hline \multicolumn{1}{|c|}{ País 1 } & País 2 & Número de artigos \\
\hline Cingapura & Índia & 1 \\
\hline EUA & Tailândia & 1 \\
\hline
\end{tabular}

Fonte: Elaborada pelos autores.

Isto pode indicar um interesse de aproximação das instituições orientais com o intuito de desenvolver e potencializar a formação de seus pesquisadores com base nos modelos de gestão descentralizados ocidentais. Vale destacar que esta assunção requer validação qualitativa ou quantitativa por meio de pesquisas futuras.

\subsection{Abordagem prática dos artigos e implicações das HMT no contexto da pesquisa}

A revisão da literatura permitiu identificar as implicações principais das tecnologias híbridas no contexto da manutenção. Os benefícios identificados quanto à sua aplicabilidade para reparo e remanufatura de peças são:

(i) Remanufatura $(R m)$ e reparo $(R)$ de componentes de alto valor (indústria aeroespacial e automotiva), com baixo custo, maior rapidez e com menos desperdiço (Despeisse \& Ford, 2015). Determinados tipos de materiais de maior resistência (incluindo compósitos) podem ser adicionados em determinadas regiões de uma peça onde ocorre maior desgaste por tempo e uso, na quantidade exata, e este material depositado pode posteriormente ser usinado para atingir os perfis desejados;

(ii) Redução do inventário de peças de reposição, aplicando, para o reparo e remanufatura, os princípios do modelo de "produção-sob-encomenda" em contraposição ao tradicional "produção-para-estocar" (make-to-order vs. make-to-stock) (Ford \& Despeisse, 2016), o que pode ser entendido como um modelo de repair/remanufacture-to-order (Rep/Rem-to-order). Rep/Rem-to-order implica que a descentralização da organização do sistema de manutenção pode alocar recursos (impressoras 3D, mão de obra, materiais, etc.) diretamente no setor operacional, ou ainda aplicando uma organização híbrida do sistema de manutenção (Velázquez et al., 2018; Tasé-Velázquez et al., 2020). Este modelo de assistência é mais factível para componentes atualizáveis, modulares e orientados ao PSS (Product-Service System) (Mastrapa et al., 2020); 
(iii) Extensão da vida útil $(E v u)$ do produto por meio de abordagens técnicas como reparo, remanufatura e recondicionamento, garantindo padrões socioeconômicos mais sustentáveis e relações mais estreitas entre produtores e consumidores (Kohtala, 2015). Melhores relações entre produtores e consumidores se dá a partir da prática sistematizada da adoção de modelos de negócios voltados à servitização como estratégia empresarial para atender aos requisitos do mercado sem gerar custos adicionais para a empresa (Hernández-Mastrapa et al., 2020), convertendo o consumidor em usuário. A Evu permite economizar capital monetário a partir da diminuição da necessidade do uso de materiais de custo elevado para fabricação de novos produtos, peças ou componentes;

(iv) Reconfiguração da cadeia de valor (Rcv) por meio da adoção das tecnologias avançadas de manufatura e seu vínculo à indústria 4.0, web 2.0, o que implicaria no futuro o surgimento de cadeias de valor mais curtas, localizadas, colaborativas e mais sustentáveis, de acordo com Gebler et al. (2014) e Strange e Zucchella (2017). A Rcv implica ademais a redução da complexidade da cadeia de suprimento e aumento da flexibilidade dos serviços logísticos (diminuição/eliminação de custos de transporte e de armazenagem de produtos terminados); customização/personalização em massa (mass customization/personalization); descentralização da manufatura; eficiência no uso de recursos e sustentabilidade, entre outros (Velázquez et al., 2020). A reconfiguração de um sistema de organização da manutenção descentralizado ou híbrido também é um elemento a ser considerado neste processo.

A análise de conteúdo dos artigos selecionados segundo a abordagem prática neles discutida, no que se refere a reparo $(R)$, remanufatura $(R m)$, extensão da vida útil ( $E v u)$ e reconfiguração da cadeia de valor $(R c v)$, é apresentada no Quadro 4. 
Quadro 4 - Abordagens práticas dos artigos

Autores

Capello et al. (2005); Capello

\& Previtali (2006); Cottam \&

Brandt (2011); Leunda et al.

(2011); Petrat et al. (2016)

Despeisse \& Ford (2015);

Ford \& Despeisse (2016)

Zhu et al. (2013); Flynn et al. (2016); Chong et al. (2018)

Graf et al. (2012; 2013)

Jones et al. (2012);

Rottwinkel et al. (2014);

Wilson et al. (2014); Zhang

et al. (2018)

King et al. (2006); Rickli et al.

(2014); Le et al. (2015;

2017a; 2017b; 2017c; 2018)

manufatura / remanufatura de peças novas a partir de peças no fim da vida útil

(end-of-life parts), extensão do ciclo de vida de peças e componentes.

Liou et al. (2002); Yu et al.

Morrow et al. (2007);

Caiazzo et al. (2017)

Karunakaran et al. (2010);

Navrotsky et al. (2015); Liu

et al. (2017); Leino et al.

(2016); Wits et al. (2016);

Knofius et al. (2019)

Ren et al. (2006, 2007); Nan

et al. (2010)

Robinson \& Scott (2014);

Vartanian (2014); Dutta \&

Froes (2017)

Abordagem prática

$R$ - Análise e avaliação da influência dos fatores que condicionam o sucesso das estratégias de reparo de peças metálicas baseadas na técnica de revestimento a laser, determinação de parâmetros do processo.

Evu/Rcv - Eficiência e sustentabilidade a partir da adoção da manufatura aditiva como modelo de produção. Vantagens, desafios, e implicações da manufatura aditiva sobre a sustentabilidade em termos de inovação, modelos de negócios e a configuração de cadeias de valor.

Rcv - Aplicações e tendências da manufatura híbrida em diferentes contextos, revisão da literatura, relação com a TI.

$R$ - Deposição de metal a laser como tecnologia para reparo de peças de alumínio e aço e, manutenção e reparo de superfícies danificadas.

$R / R m / R c v$ - Sistema flexível de revestimento a laser e modelo prático de deposição direta de metal a laser para reparo e remanufatura de pás de turbinas e avaliação do ciclo de vida.

$E v u / R m / R c v$ - Comparação de estratégias para reduzir o descarte de produtos no fim da vida útil. Modelo conceitual para remanufatura de produtos no fim da vida útil (end-of-life products). Abordagem para reutilização de material na produção de novos produtos a partir de componentes já existentes, estratégia para $R$ - Análise comparativa dos processos de reparo de componentes metálicos por soldagem e deposição de metal a laser.

$R / R m$ - Estudo da técnica de deposição direta de metal para reparo e remanufatura de ferramentas e moldes e de produtos danificados.

$R / R m$ - Principais implicações da manufatura aditiva e configuração de estratégias para fabricação e reparo de estruturas complexas e componentes metálicos. Estratégias de manutenção, reparo e inspeção (MRO) para atender as necessidades específicas dos usuários finais usando AM. Análise de custos da adoção da AM.

$R / R m$ - Sistema híbrido para reparo e remanufatura de componentes para aprimorar a qualidade da superfície de peças com alto custo de aquisição. $R / R m / E v u$ - Generalidades e vantagens da manufatura aditiva. Implicações no reparo, remanufatura e extensão da vida útil de produtos em uso (in-service products) e de componentes produzidos a partir de ligas de titânio.

Fonte: Elaborado pelos autores. 
No Quadro 4, pode ser observado que em relação a reparo $(R), 69$ \% dos trabalhos tratam desse assunto; por sua vez, $64 \%, 41 \%$ e $31 \%$ tocam o tema relacionado a remanufatura $(R m)$, reconfiguração da cadeia de valor $(R c v)$ e extensão da vida útil $(E v u)$, respectivamente. A soma total da porcentagem é maior do que 100 \% já que um mesmo trabalho pode tratar de dois ou mais temas e foi incluído mais de uma vez no cálculo.

A capacidade das HMTs para reparo e remanufatura, e a subsequente extensão dos ciclos de vida do produto, criarão incentivos para que as empresas adotem modelos de negócios orientados, tanto para os serviços, quanto para os produtos (Ford \& Despeisse, 2016). Porém, introduzem como limitação a necessidade de desenvolver e certificar processos de manutenção, com reparo e remanufatura por meio do emprego das HMTs.

\section{Considerações finais}

Este artigo apresentou uma revisão sistemática da literatura sobre as implicações da manufatura híbrida no reparo e remanufatura de peças no contexto da manutenção. A metodologia usada neste artigo não revela apenas a tendência em nível macro incluindo quantidade de publicações, afiliações, autores e periódicos. Além disso, expõe, de forma breve, o conhecimento contido nesses documentos, na forma de escopo prático dos trabalhos e benefícios dos sistemas híbridos no que diz respeito a reparo $(R)$, remanufatura $(R m)$ e extensão da vida útil $(E v u)$ dos produtos e, consequentemente reconfiguração das cadeias de valor (Rcv). É pertinente observar que a produção científica na área ainda é relativamente pouca a partir da quantidade de publicações, e requer rápido avanço, dada a dinâmica volátil e de mudanças aceleradas no ambiente empresarial. A visualização das palavras-chave, assim como as abordagens práticas expostas nos artigos, revela certo interesse em pesquisar sobre impacto destas tecnologias em relação à sustentabilidade, extensão do ciclo de vida do produto e ao uso eficiente de recursos. Isso, por consequência, indica a necessidade de entender como gerenciar esses sistemas de manufatura avançados e seu vínculo a sistemas e programas de manutenção adequados, mas, nesta área de pesquisa, o foco recai em assuntos relacionados ao reparo e remanufatura. A tendência de publicação vem aumentando desde 2002; no entanto, a frequência absoluta dessas publicações ainda pode ser considerada baixa. Os periódicos onde mais se publicou foram o Physics Procedia (5 publicações), o Journal of Cleaner Production e o Procedia CIRP, com 4 artigos em cada um. Foi identificada e mapeada uma rede de colaboração entre pesquisadores de distintas nacionalidades, envolvendo 15 países dos distintos continentes. A distribuição geográfica das afiliações contribuintes na pesquisa foca-se na Europa e na América do Norte. As principais organizações colaboradoras estão nos EUA, França, Alemanha, Reino Unido, Itália e Países Baixos. A base de dados em destaque, considerando a quantidade de artigos levantada é ScienceDirect, com 18 artigos. 
Como qualquer outro estudo, este trabalho também apresenta certas limitações. Além de artigos de periódicos, também poderiam ter sido incluídos no estudo outros recursos disponíveis fora de bases de dados acadêmicas, e livros para análises adicionais. Também poderia ter-se incluído na análise bibliométrica os autores com maior quantidade de citação, co-citação, natureza dos trabalhos e interdependência entre as palavras-chave. Os resultados deste trabalho têm implicações acadêmicas e práticas. O conjunto atual de resultados sobre a literatura mapeada sobre os benefícios do uso das HMT na manutenção, com reparo e remanufatura permite afirmar que é um campo relativamente novo e poderá contribuir na expansão do desenvolvimento desta área, aprofundando na inclusão destas tecnologias no desenvolvimento e implantação de atividades e sistemas de manutenção.

\section{Agradecimentos}

O presente trabalho foi realizado com apoio da Coordenação de Aperfeiçoamento de Pessoal de Nível Superior - Brasil (CAPES) - Código de Financiamento 001.

\section{Referências}

ABNT - Associação Brasileira de Normas Técnicas. (1994). ABNT NBR 5462 - Confiabilidade e Mantenabilidade. ABNT/CB-003 Eletricidade, 37.

Attaran, M. (2017). The rise of 3-D printing: The advantages of additive manufacturing over traditional manufacturing. Business Horizons, (60), 677-688.

Caiazzo, F., Alfieri, V., Argenio, P., \& Sergi, V. (2017). Additive manufacturing by means of laser-aided directed metal deposition of 2024 aluminium powder: Investigation and optimization. Advances in Mechanical Engineering, 9(8), 1-12.

Capello, E., \& Previtali, B. (2006). The influence of operator skills, process parameters and materials on clad shape in repair using laser cladding by wire. Journal of Materials Processing Technology, 174, 223-232.

Capello, E., Colombo, D., \& Previtali, B. (2005). Repairing of sintered tools using laser cladding by wire. Journal of Materials Processing Technology, 164-165, 990-1000.

Capes - Coordenação de Aperfeiçoamento de Pessoal de Nível Superior. Disponível em http://wwwperiodicos-capes-gov-br.ez100.periodicos.capes.gov.br/ Acesso: fevereiro 2019.

Chong, L., Ramakrishna, S., \& Singh, S. (2018). A review of digital manufacturing-based hybrid additive manufacturing processes. International Journal of Advanced Manufacturing Technology, 95, 22812300.

Cottam, R., \& Brandt, M. (2011). Laser cladding of Ti-6Al-4V powder on Ti-6Al-4V substrate: Effect of laser cladding parameters on microstructure. In: Physics Procedia, 12, 323-329.

Dasgupta, J. L., \& Dinda, A. K. (2014). A descriptive framework for additive remanufacturing systems. International Journal of Rapid Manufacturing, 4(2/3/4), 199-218. 
Despeisse, M., \& Ford, S. (2015). The role of additive manufacturing in improving resource efficiency and sustainability. In: IFIP Advances in Information and Communication Technology, (3), ISSN 20588887.

Dutta, B., \& Froes, S. (2017). The Additive Manufacturing (AM) of titanium alloys. Metal Powder Report, 72(2), 96-106.

Fahimnia, B., Tang, C., Davarzani, H. \& Sarkis, J. (2015). Quantitative models for managing supply chain risks: a review. European Journal of Operational Research, 247(1), 1-15.

Faludi, J., Bayly, C., Bhogal, S., \& Iribarne, M. (2015). Comparing environmental impacts of additive manufacturing vs. traditional machining via life-cycle assessment. Rapid Prototyping Journal, 21(1), 14-33.

Flynn, J. M., Shokrani, A., Newman, S. T., \& Dhokia, V. (2016). Hybrid additive and subtractive machine tools - Research and industrial developments. International Journal of Machine Tools and Manufacture, (101), 79-101.

Ford, S., \& Despeisse, M. (2016). Additive manufacturing and sustainability: an exploratory study of the advantages and challenges. Journal of Cleaner Production, 137, 1573-1587.

Graf, B., Ammer, S., Gumenyuk, A., \& Rethmeier, M. (2013). Design of experiments for laser metal deposition in maintenance, repair and overhaul applications. In: Procedia CIRP, 11, 245-248.

Graf, B., Gumenyuk, A., \& Rethmeier, M. (2012). Laser metal deposition as repair technology for stainless steel and titanium alloys. In: Physics Procedia, 83, $761-768$.

Guessasma, S., Zhang, W., Zhu, J., Belhabib, S., \& Nouri, H. (2015). Challenges of additive manufacturing technologies from an optimization perspective. International Journal for Simulation and Multidisciplinary Design Optimization, 6, (A9), 1-13.

Hernández-Mastrapa, L., Pontes-de Assumpção, M. R., \& Tasé-Velázquez, D. R. (2020). Análisis bibliográfico sobre toma de decisiones en la servitización. EmTHYMós, Revista de Estudios Empresariales, 1(1), 21-34.

Ida - Institute for Defense Analyses. (2012). Emerging global trends in advanced manufacturing. IDA Paper P-4603. EE.UU, 248.

Ijomah, W. (2002). A model-based definition of the generic remanufacturing business process. Doctoral Thesis. University of Plymouth, USA.

Jones, J., Mcnutt, P., Tosi, R., Perry, C., \& Wimpenny, D. (2012). Remanufacture of turbine blades by laser cladding, machining and in-process scanning in a single machine. $23^{\text {rd }}$ Annual International Solid Freeform Fabrication Symposium, Austin, Texas, USA, 821-827.

Karunakaran, K., Suryakumar. S, Pushpa, V., \& Akula, S. (2010) Low cost integration of additive and subtractive processes for hybrid layered manufacturing. Robotics and Computer-Integrated Manufacturing, 26(5), 490-499.

King, A. M., Burgess, S. C., ljomah, W., \& McMahon, C. A. (2006). Reducing waste: Repair, recondition, remanufacture or recycle? Sustainable Development, 14, 257-267. 
Knofius, N., van der Heijden, M. C., \& Zijm, W. H. M. (2019). Consolidating spare parts for asset maintenance with additive manufacturing. International Journal of Production Economics, 208(November 2018), 269-280.

Kohtala, C., \& Hyysalo, S. (2015). Anticipated environmental sustainability of personal fabrication. Journal of Cleaner Production, 99, 333-344.

Lasemi, A., Xue, D., \& Gu, P. Recent development in CNC machining of freeform surfaces: a state-ofthe-art review. Computer-Aided Design, 42, 641-54.

Le, V. T., Paris, H., \& Mandil, G. (2015). Using additive and subtractive manufacturing technologies in a new remanufacturing strategy to produce new parts from End-of-Life parts. $22^{\text {nd }}$ French Congress of Mechanics, Lyon, France, 1-8.

Le, V. T., Paris, H., \& Mandil, G. (2017b). Process planning for combined additive and subtractive manufacturing technologies in a remanufacturing context. Journal of Manufacturing Systems, 44, 243-254.

Le, V. T., Paris, H., \& Mandil, G. (2017c). Environmental impact assessment of an innovative strategy based on an additive and subtractive manufacturing combination. Journal of Cleaner Production, 164, 508-523.

Le, V. T., Paris, H., \& Mandil, G. (2018). Extracting features for manufacture of parts from existing components based on combining additive and subtractive technologies. International Journal on Interactive Design and Manufacturing (IJIDeM), 12, 525-536.

Le, V. T., Paris, H., Mandil, G., \& Brissaud, D. (2017a). A direct material reuse approach based on additive and subtractive manufacturing technologies for manufacture of parts from existing components. In: Procedia CIRP, 61, 229-234.

Leino, M., Pekkarinen, J., \& Soukka, R. (2016). The role of laser additive manufacturing methods of metals in repair, refurbishment and remanufacturing - Enabling circular economy. Physics Procedia, 83, 752-760.

Leunda, J., Soriano, C., Sanz, C., \& Navas, V. G. (2011). Laser cladding of vanadium-carbide tool steels for die repair. In: Physics Procedia, 12, 345-352.

Liou, F., Wang, J., Prakash, S., \& Joshi, Y. (2002). Laser Aided Part Repair-A Review. Proceedings ASME International Manufacturing Science and Engineering Conference (MSEC), 57-64.

Liu, R., Wang, Z., Sparks, T., Liou, F., \& Nedic, C. (2017). Stereo vision-based repair of metallic components. Rapid Prototyping Journal, 23(1), 65-73.

Mastrapa, L. H., Assumpção, M. P., Tasé Velázquez, D. R., Gennaro, C. K., \& de Oliveira, E. D. (2020). Product-Service System Modularization: A Systematic Review. In: Anisic Z., Lalic B., Gracanin D. (eds) Proceedings on 25th International Joint Conference on Industrial Engineering and Operations Management - IJCIEOM. IJCIEOM 2019. Lecture Notes on Multidisciplinary Industrial Engineering. Springer, Cham.

Miguel, P. A. C. (2012). Metodologia de pesquisa para engenharia de produção e gestão de operações. 2da Ed. - Rio de Janeiro: Elsevier: ABEPRO, ISBN 978-85-352-4850-0.

Morrow, W. R., Qi, H., Kim, I., Mazumder, J., \& Skerlos, S. J. (2007). Environmental aspects of laserbased and conventional tool and die manufacturing. Journal of Cleaner Production, 15, 932-943. 
Nan, L., Liu, W., \& Zhang, K. (2010). Laser remanufacturing based on the integration of reverse engineering and laser cladding. International Journal of Computer Applications in Technology, 37(2), 116-124.

Navrotsky, V., Graichen, A., \& Brodin, H. (2015). Industrialization of 3D printing (additive manufacturing) for gas turbine components repair and manufacturing. VGB PowerTech, 12, 48-52.

Newman, S. T., Zhu, Z., Dhokia, V., \& Shokrani, A. (2016). Process planning for additive and subtractive manufacturing technologies. CIRP Annals - Manufacturing Technology, (64), 467-470.

Petrat, T., Graf, B., Gumenyuk, A., \& Rethmeier, M. (2016). Laser metal deposition as repair technology for a gas turbine burner made of inconel 718. In: Physics Procedia, 83, 761-768.

Pinto, K., \& Xavier, J. N. (2009). Manutenção: função estratégica. 3ạ ed. Rio de Janeiro. Qualitymark: Petrobrás, 384.

Ren, L., Eiamsa-Ard, K., Ruan, J., Liou, F. (2007). Part repairing using a hybrid manufacturing system. Proceedings of International Manufacturing Science and Engineering Conference, Atlanta, Georgia, USA, 1-8.

Ren, L., Padathu, A. P., Ruan, J., Sparks, T., \& Liou, F. W. (2006). Three dimensional die repair using a hybrid manufacturing system. Proceedings of Solid Freeform Fabrication Symposium. Austin, TX, EUA, 51-59.

Rickli, J. L., Dasgupta, J. L., Dinda, A. K. (2014). A descriptive framework for additive remanufacturing systems. International Journal of Rapid Manufacturing, 4, (2/3/4), 199-218.

Robinson, L., \& Scott, J. (2014). Layers of complexity: making the promises possible for additive manufacturing of metals. Journal of the Minerals, Metals, and Materials Society, 66(11), 2197-2207.

Rottwinkel, B., Nölke, C., Kaierle, S., \& Wesling, V. (2014). Crack repair of single crystal turbine blades using laser cladding technology. In: Procedia CIRP, 22, 263-267.

Singh, S., Ramakrishna, S., \& Singh, S. (2017). Issues in additive manufacturing: A review. Journal of Manufacturing Processes, (25), 185-200.

Tasé-Velázquez, D. R., Camello-Lima, C. R., \& Hernández-Mastrapa, L. (2020). Modelo para la gestión del mantenimiento de un sistema de fabricación híbrido con base en políticas corporativas y de producción. EmTHYMÓS, Revista de Estudios Empresariales, 1(2), 118-134.

Tofail, S. A. M., Koumoulos, E. P., Bandyopadhyay, A., Bose, S., O’Donoghue, L., \& Charitidis, C. (2018). Additive manufacturing scientific and technological challenges, market uptake and opportunities. Materials Today, 21(1), 22-37.

Vartanian, K. (2014). 3D Printing using powder metals choosing the right process for the right application. Materials \& Assembly, 44-51.

Velázquez, D. R. T., Lima, C. R. C., Helleno, A. L., Mastrapa, L. H., Oliveira, E. D., \& Gennaro, C. K. (2018). Esquema conceitual para gerenciamento da manutenção no contexto das tecnologias híbridas de manufatura. BTSym 2018 Proceedings, 1, 1-8.

Velázquez, D. R. T., Simon, A. T., Helleno, A. L., \& Mastrapa, L. H. (2020). Implications of additive manufacturing on supply chain and logistics. Independent Journal of Management \& Production, 11(4), 1279-1302. 
Wilson, J. M., Piya, C., Shin, Y. C., Zhao, F., \& Ramani, K. (2014). Remanufacturing of turbine blades by laser direct deposition with its energy and environmental impact analysis. Journal of Cleaner Production, 80, 170-178.

Wits, W. W., García, J. R. R., \& Becker, J. M. J. (2016). How Additive Manufacturing Enables more Sustainable End-user Maintenance, Repair and Overhaul (MRO) Strategies. Procedia CIRP, 40, 693698.

Xiao, Z.; Yang, Y.; Xiao, R.; Bai, Y.; Song, C.; Wang, D. (2018). Evaluation of topology-optimized lattice structures manufactured via selective laser melting. Materials \& Design, 143, 27-37.

Yu, J. H., Choi, Y. S., Shim, D. S., \& Park, S. H. (2018). Repairing casting part using laser assisted additive metal-layer deposition and its mechanical properties. Optics and Laser Technology, 106, 87-93.

Zębala, W., \& Plaza, M. Comparative study of 3- and 5-axis CNC centers for free-form machining of difficult-to-cut material. International Journal of Production Economics, 158, 345-358.

Zhang, X., Li, W., Cui, W., \& Liou, F. (2018). Modeling of worn surface geometry for engine blade repair using Laser-aided Direct Metal Deposition process. Manufacturing Letters, 15PA, 1-4.

Zhu, Z., Dhokia, V., Nassehi, A., \& Newman, S. T. (2013). A review of hybrid manufacturing processesstate of the art and future perspectives. International Journal of Computer Integrated Manufacturing, 26(7), 596-615.

Recebido em: 14 mar. 2019 / Aprovado em: 09 out. 2019

Para referenciar este texto

American Psychological Association (APA)

Velázquez, D. R. T., Helleno, A. L., Lima, C. R. C., \& Mastrapa, L. H. (2020, out./dez.). Implicações das tecnologias híbridas de fabricação nos processos de reparo e remanufatura de peças no contexto da manutenção: uma revisão sistemática. Exacta, 18(4), 778-798. https://doi.org/10.5585/exactaep.v18n4.13270. 